\title{
Dupilumab Induced Limbal Stem Cell Deficiency
}

\author{
Urmi Mehta ${ }^{1,2}$ \\ Marjan Farid ${ }^{\prime}$
}

'Gavin Herbert Eye Institute, Department of Ophthalmology, University of California, Irvine, CA, 92697, USA; ${ }^{2}$ Western University of Health Sciences, Pomona, CA, 91766, USA
Correspondence: Marjan Farid

Gavin Herbert Eye Institute, University of

California, Irvine, CA, USA

Tel + I 949 824-2020

Email mfarid@hs.uci.edu

\begin{abstract}
Dupilumab is a monoclonal antibody that is used for the treatment of atopic dermatitis (AD) in adults. However, increasing reports of ocular complications including conjunctivitis and dry eye disease have been documented. In this report, we describe a case of a patient who developed limbal stem cell deficiency (LSCD) after prolonged Dupilumab use. A 56-year-old Caucasian male with a history of AD presented with gradual onset cloudy vision and extensive diffuse symblepharon resulting from Dupilumab treatment. He was diagnosed with cicatrizing blepharoconjunctivitis and secondary LSCD after slit lamp examination. In conclusion, LSCD secondary to cicatricial disease is a severe adverse ocular complication caused from long-term Dupilumab treatment.
\end{abstract}

Keywords: limbal stem cell disease, symblepharon, cicatrizing blepharoconjunctivitis, Dupilumab, dupixent

\section{Introduction}

Dupilumab is a human monoclonal antibody that works by inhibiting interleukin (IL)-4 and IL-13 signaling by binding to the shared IL-4 alpha subunit. ${ }^{1,2}$ It is efficacious in treating moderate to severe atopic dermatitis (AD) in adults whose disease has been refractory to or inadequately managed by other immunosuppressive agents. ${ }^{3}$

The most common adverse ophthalmic complication from Dupilumab treatment is conjunctivitis. ${ }^{4,5}$ Although most cases of conjunctivitis are mild and self-limiting, few cases have required drug cessation due to severe ocular surface disease. ${ }^{6}$ Other reported adverse reactions include eye irritation, blepharitis, ${ }^{7}$ blepharoconjunctivitis, ${ }^{6,8}$ periocular dermatitis, ${ }^{6}$ cicatricial ectropion, ${ }^{6,8}$ keratitis, ${ }^{7,8}$ limbitis, ${ }^{9}$ and limbal stem cell deficiency (LSCD). ${ }^{3}$

In this report, we present a case of a healthy 56-year-old male who developed bilateral LSCD with extensive diffuse symblepharon affecting his extraocular movements after prolonged Dupilumab therapy for AD.

\section{Case Presentation}

A 56-year-old Caucasian male with a history of AD, LASIK OU (2000), trauma induced corneal flap removal (2010) and SK of the left eye (2020) presented to the ophthalmology clinic with bilateral "shrinking of his eyes" that gradually developed after starting Dupilumab treatment. He was started on bimonthly Dupilumab injections (300 mg SC q2wk) in January 2015. About one year after starting the medication, he started noticing ocular complications, including peeling and burning of his eyelid margins (360 degree involvement) that extended beyond the palpebral fissures, clouding and blurring of his vision, and trichiasis. Symptoms gradually worsened, and by 

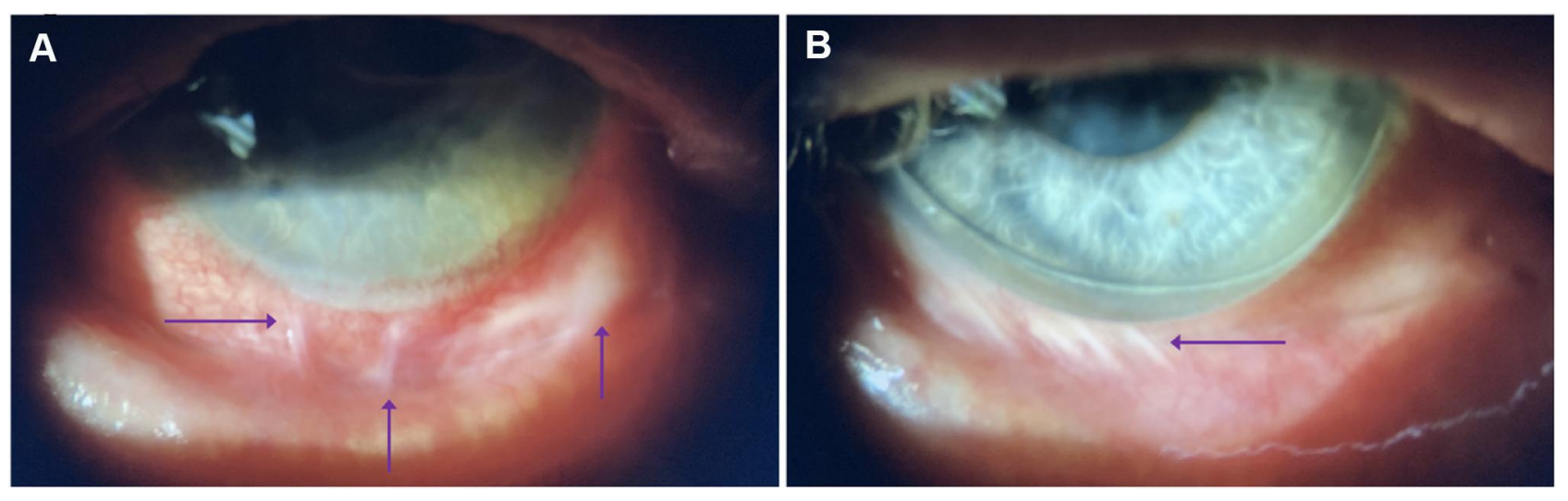

Figure I Arrows point to symblepharon formation seen inferiorly in the right (A) and left (B) eyes.

2018, he developed diffuse bilateral symblepharon that led to gaze restriction in extreme lateral gazes and forniceal shortening causing his scleral lens to no longer fit.

He discontinued Dupilumab in September 2019 due to worsening ocular complications, including double vision in extreme lateral gaze, worsening vision of both eyes, and chronic eyelid and ocular surface inflammation. After discontinuation of the Dupilumab, he reported a gradual improvement in his vision and skin around his eyelids.

On physical exam, BCVA at distance with RGP lenses was 20/30 OU. Slit lamp exam showed bilateral eyelid edema, inflammation, and extensive severe symblepharon throughout the inferior, nasal, and temporal regions (Figure 1), causing restriction of extreme lateral gaze and forniceal shortening. Anterior corneal scarring with subepithelial haze, obscured limbal architecture, and poor corneal epithelial cell health with whorled pattern epitheliopathy was seen bilaterally (Figure 2A and B). However, the left eye was more severe, showing more

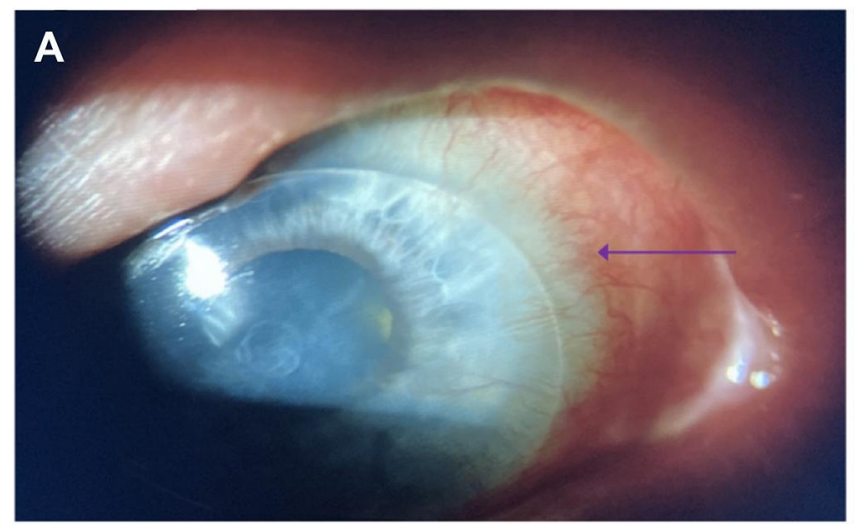

Figure 2 Limbal stem cell deficiency in the right $(\mathbf{A})$ and left $(\mathbf{B})$ eye. Arrows point to neovascularization and conjunctivalization of the cornea. Whorled keratopathy, obscured limbal architecture, subepithelial haze, and conjunctival hyperemia are also present bilaterally.

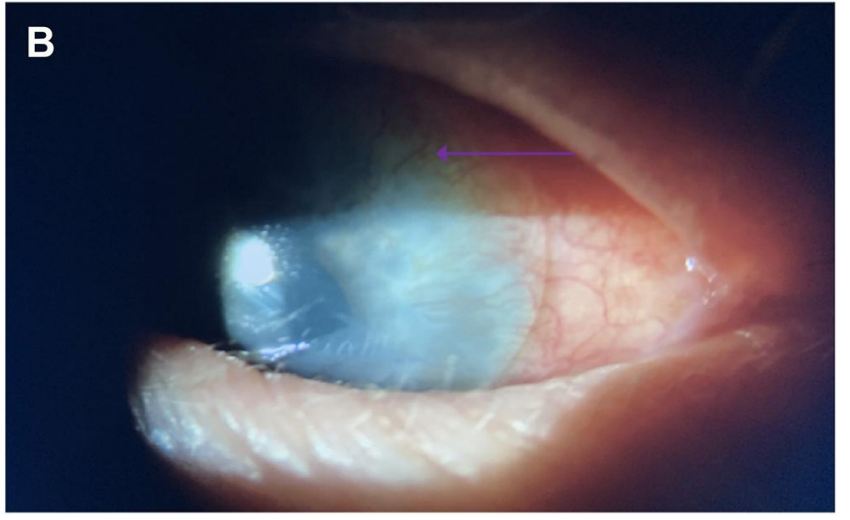

corneal neovascularization and temporal conjunctivalization of cornea (Figure 2B).

He was started on autologous serum eyedrops $75 \%$ (q2hour) to be used in conjunction with fluorometholone $0.1 \%$ (BID), which he had been using prior to his visit to the clinic. Further topical immunomodulators such as cyclosporine or lifitegrast will be added to minimize ocular inflammation. He is also being closely monitored for progression as systemic immunosuppression may be required as well.

\section{Discussion}

Our patient presented with bilateral diffuse LSCD after chronic Dupilumab therapy for AD. Only one other report documents this rare adverse complication in a patient with a history of pellucid marginal degeneration who discontinued Dupilumab 12 weeks after initiating therapy due to the development of LSCD. Our patient was on Dupilumab therapy for 57 months before discontinuing, which could explain the severity of his ocular manifestations. 
Dupilumab exerts its effects by binding to the IL-4 alpha subunit, preventing IL-4 and IL-13 signaling. In mice studies, IL-13 has been shown to stimulate conjunctival goblet cell production. Goblet cells regulate inflammation, stimulate mucin production, and maintain the overall health of the ocular surface epithelium. ${ }^{10}$ Therefore, blocking IL-13 signaling would theoretically lead to goblet cell hypoplasia, resulting in increased ocular inflammation, decreased mucin production, tear film instability, and mucosal epithelial barrier dysfunction. ${ }^{7,11}$ A recent study published by Bakker et al confirmed this finding in humans. In this study, conjunctival biopsies taken from patients who developed new or worsening conjunctivitis after treatment with Dupilumab for at least 16 weeks showed decreased intraepithelial mucuscontaining goblet cells compared to the control group. ${ }^{11}$ A separate study published by Barnett and Afshari (2020) evaluated mucin 5ac (Muc5AC), a specific marker of goblet cells, in patients treated with Dupilumab. Compared with the control group, patients taking Dupilumab showed decreased mucin production and decreased or absent goblet cells on conjunctival biopsies. Subjects reported a significantly increased occurrence of ocular fatigue, pain, redness, and pruritus and were diagnosed with varying degrees of conjunctivitis, keratitis, and blepharitis. $^{7}$

In addition to goblet cell loss, Dupilumab associated conjunctivitis has been hypothesized to be due to heightened OX40 ligand activity from IL-4 and IL-13 signaling inhibition, eosinophilia, and increased Demodex infestation due to changes in the ocular surface environment. ${ }^{6-8,12}$ Ocular surface inflammation has been shown to develop 1 to 8 months after initiating Dupilumab therapy, with an average onset of 3.5 months. ${ }^{9}$ A prior study showed that those who developed mild conjunctivitis were adequately treated with warm compresses and preservative free artificial tears. Those who developed severe follicular conjunctivitis often had a history of allergic conjunctivitis that was either triggered or worsened by Dupilumab treatment. ${ }^{9}$ Blepharitis, conjunctival hyperemia, and limbitis often developed in these patients, which resolved with either steroid treatment or discontinuation of Dupilumab. ${ }^{9}$ Steroid treatment ${ }^{5}$ using either prednisolone $0.5 \%$, fluorometholone $0.1 \%$, or dexamethasone $0.1 \%$ and cyclosporine $0.05 \%$ has been the mainstay of therapy. ${ }^{6}$ Cyclosporine is a calcineurin inhibitor that has the potential to increase goblet cell numbers by inhibiting T-cell mediated immune responses. ${ }^{6}$
Recent studies demonstrated a subset of patients with severe conjunctivitis ${ }^{12}$ and limbitis ${ }^{5}$ that did not improve with anti-inflammatory treatment. Severe chronic inflammation can stress limbal stem cells, leading to LSCD. It is reasonable to have patients see their eye care provider before initiating treatment since a large percentage of patients with AD considering Dupilumab treatment have undiagnosed ocular surface inflammation. ${ }^{6}$ Ocular surface disease can therefore be treated aggressively before initiation of Dupilumab. These patients should also have regular follow-ups with their eye care provider especially if they begin noticing new onset or worsening vision, ocular pain, redness, burning, and dryness to prevent long-term irreversible complications from the medication.

Risk factors for developing Dupilumab-induced ocular surface disease include severe $\mathrm{AD}$ at baseline and a prior history of conjunctivitis. ${ }^{6,8,12}$ At baseline, our patient was reported to have severe AD. He noticed ocular irritation and discomfort 12 months after starting therapy. In addition to developing chronic blepharoconjunctivitis, he developed diffuse cicatrizing conjunctival disease that led to extensive symblepharon and forniceal shortening, indicative of severe goblet cell loss. He also developed severe chronic ocular surface and periocular inflammation, leading to limbal architecture loss, poor corneal epithelial cell health with whorled keratopathy, and subepithelial haze, consistent with limbal stem cell burnout and LSCD. His symptoms did improve after discontinuing the medication. However, the long-term effects from limbal stem cell deficiency and cicatrizing symblepharon are irreversible sequelae that need life-long management and care.

\section{Conclusion}

Dupilumab can cause adverse ocular complications ranging from conjunctivitis to LSCD. As a result, patients should be monitored routinely before irreversible damage occurs.

\section{Patient Consent}

Consent to publish this case report has been obtained from the patient(s) in writing.

Institutional approval was not required to publish the case details.

\section{Funding}

The authors acknowledge departmental support from an RPB unrestricted grant. 


\section{Disclosure}

The authors have no financial or other conflicts of interest.

\section{References}

1. Ivert LU, Wahlgren C, Ivert L, et al. Eye complications during dupilumab treatment for severe atopic dermatitis. Acta Derm Venereol. 2019;99(4):375-378. doi:10.2340/00015555-3121

2. Albader SS, Alharbi AA, Alenezi RF, et al. Dupilumab side effect in a patient with atopic dermatitis: a Case Report Study. Biologics. 2019;13:79-82. doi:10.2147/BTT.S195512

3. Ariens LFM, Schaft J, Bakker DS, et al. Dupilumab is very effective in a large cohort of difficult-to-treat adult atopic dermatitis patients: first clinical and biomarker results from the BioDay registry. Allergy. 2020;75(1):116-126. doi:10.1111/all.14080

4. Fukuda K, Ishida W, Kishimoto T, et al. Development of conjunctivitis with a conjunctival proliferative lesion in a patient treated with dupilumab for atopic dermatitis. Allergol Int. 2019;68(3):383-384. doi:10.1016/j.alit.2018.12.012

5. Achten R, Bakker D, Ariens L, et al. Long-term follow-up and treatment outcomes of conjunctivitis during dupilumab treatment in patients with moderate-to-severe atopic dermatitis. J Allergy Clin Immunol Pract. 2021;9(3):1389-1392.e2. doi:10.1016/j. jaip.2020.09.042
6. Popiela MZ, Barbara R, Turnbull AMJ, et al. Dupilumab-associated ocular surface disease: presentation, management and long-term sequelae. Eye. 2021. doi:10.1038/s41433-020-01379-9

7. Barnett BP, Afshari NA. Dupilumab-associated mucin deficiency (DAMD). Transl Vis Sci Technol. 2020;9(3):29. doi:10.1167/ tvst.9.3.29

8. Nahum Y, Mimouni M, Livny E, et al. Dupilumab-induced ocular surface disease (DIOSD) in patients with atopic dermatitis: clinical presentation, risk factors for development and outcomes of treatment with tacrolimus ointment. Br J Ophthalmol. 2019;104(6):776-779. doi:10.1136/bjophthalmol-2019-315010

9. Maudinet A, Law-Koune S, Duretz C, et al. Ocular surface diseases induced by dupilumab in severe atopic dermatitis. Ophthalmol Ther. 2019;8(3):485-490. doi:10.1007/s40123-019-0191-9

10. Tukler Henriksson J, Coursey TG, Corry DB, et al. IL-13 stimulates proliferation and expression of mucin and immunomodulatory genes in cultured conjunctival goblet cells. Invest Ophthalmol Vis Sci. 2015;56(8):4186-4197. doi:10.1167/iovs.14-15496

11. Bakker DS, Ariens LFM, Luijk C, et al. Goblet cell scarcity and conjunctival inflammation during treatment with dupilumab in patients with atopic dermatitis. $B r \quad J$ Dermatol. 2019;180 (5):1248-1249. doi:10.1111/bjd.17538

12. Treister AD, Kraff-Cooper C, Lio PA. Risk factors for dupilumab-associated conjunctivitis in patients with atopic dermatitis. JAMA Dermatol. 2018;154(10):1208-1211. doi:10.1001/ jamadermatol.2018.2690

\section{Publish your work in this journal}

The International Medical Case Reports Journal is an international, peer-reviewed open-access journal publishing original case reports from all medical specialties. Previously unpublished medical posters are also accepted relating to any area of clinical or preclinical science. Submissions should not normally exceed 2,000 words or 4 published pages including figures, diagrams and references. The manuscript management system is completely online and includes a very quick and fair peer-review system, which is all easy to use. Visit http://www.dovepress.com/testimonials.php to read real quotes from published authors. 